\title{
SURGERY \\ Good outcomes with low-energy direct current prostate ablation
}

A new canine study has demonstrated excellent results in terms of postoperative erectile function and nerve preservation using the experimental technique of low-energy direct current (LEDC) focal ablation. "Most ablative technologies that have been studied so far rely on thermal energy, including cryoablation and high-intensity focused ultrasonography (HIFU)," explains investigator Matvey Tsivian at the Duke University Medical Center, Durham, NC. By contrast, LEDC involves the delivery of pulses of electric current into the tissue, a process that creates pores in cell membranes. This electroporation results in cell death without concomitant extreme changes in temperature associated with other ablative techniques.

Although LEDC ablation has previously been shown in vitro and in vivo (in a canine model) to be effective in terms of its ability to kill prostate cancer cells and maintain distinct margins around the prostate, Tsivian and his colleague Thomas Polascik, Director of the focal therapy programme at the Duke Cancer Institute, needed to assess the functional outcomes after prostate ablation if the technique was to considered a plausible rival to existing techniques. They treated 12 dogs (median prostate volume of $12.1 \mathrm{ml}$, range $8.9-17.3 \mathrm{ml}$ ) using the NanoKnife ${ }^{\mathrm{TM}}$ (AngioDynamics, Queensbury, NY) LEDC device, under the guidance of transrectal ultrasonography, and assessed the postprocedural outcomes of the animals.

No intraoperative complications occurred. Haematuria, which was observed in 10 dogs, spontaneously resolved within 3 days and urinary retention was not recorded in any of the animals. "Most relevant to prostate ablation is the nerve tissue, which has a pivotal role in preservation of erectile function," explains Tsivian. The animals were divided into two groups of equal size $(n=6)$ for final pathological evaluation 7 days and 27-28 days after the procedure. In the first group, all the animals were able to achieve erection within 5 days; all of those in the second group were able to achieve erection at 24-25 days. "LEDC, unlike thermal ablation, seems to preserve nervous tissue in a selective manner and might achieve better preservation of erectile function."

At the final histopathological examination, the specimens from the animals in the first group showed signs of necrosis, haemorrhage and inflammation in the ablation zone. However, by days 27-28, the zone had been replaced with fibrous connective tissue. Most importantly, no injuries to the urethra, cavernous nerves, rectal wall or prostatic capsule were noted in any of the animals, which bodes extremely well for the technique as a future treatment for prostate cancer and BPH. "Our study suggests that tissue injury is very controllable and predictable whereby very selective tissue destruction can be achieved without extending the damage to adjacent structures," concludes Tsivian. Is clinical evaluation the next step for LEDC?

Mina Razzak

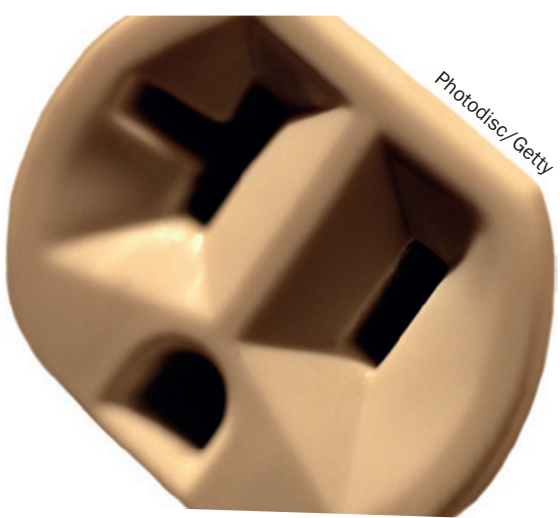

Original article Tsivian, M. \& Polascik, T. J. Bilateral focal ablation of prostate tissue using low energy

direct current (LEDC): a preclinical canine study. BJU Int. doi:10.1111/bju.12227 